\title{
Adaptação transcultural do questionário VEINES/QOL-SYM: avaliação da qualidade de vida e sintomas na doença venosa crônica
}

\author{
Transcultural Adaptation of VEINES/QOL-Sym Questionnaire: Evaluation of Quality of life and \\ Symptoms in Chronic Venous Disease
}

Regina Márcia Faria de Moura', Gabriela Silva Gonçalves², Túlio Pinho Navarro³, Raquel Rodrigues Britto ${ }^{4}$, Rosângela Correa Dias ${ }^{5}$

\begin{abstract}
Resumo
Objetivo: Adaptar transculturalmente e analisar as propriedades clinimétricas do VEINES-QOLSym, um questionário específico para avaliar a qualidade de vida e os sintomas na doença venosa crônica.

Métodos: O VEINES-QOLSym foi adaptado segundo metodologia recomendada e aplicado em 74 indivíduos (53,7士12,3 anos) com doença venosa crônica, assistidos ambulatorialmente.

Resultados: Os resultados evidenciaram boa consistência interna do VEINES-QOL (alfa de Cronbach - $\alpha=0,88$ ) e do VEINES/Sym ( $\alpha=0,84$ ). A confiabilidade inter e intra-examinadores avaliadas pelo coeficiente de correlação intraclasse variou de 0,95-0,98 para o VEINES-QOL e de 0,76-0,79 para o VEINES/Sym. O coeficiente de correlação intraclasse foi calculado para cada item e alguns apresentaram valores abaixo do esperado merecendo revisão. As correlações com SF-36 foram significativas ( $p<0,001)$, porém com moderada magnitude. Apenas o VEINES-QOL foi capaz de distinguir os indivíduos em dois grupos, de acordo com a gravidade da doença venosa crônica $(p=0,02)$.

Conclusões: O VEINES-QOL/Sym, versão português-Brasil, apresentou boas propriedades clinimétricas e demonstrou ser aplicável na população.
\end{abstract}

Palavras-chave: Tradução; psicometria; questionários; insuficiência venosa; qualidade de vida.

\begin{abstract}
Objective: To adapt cross-culturally and to analyze the clinimetric properties of the VEINES-QOLSym, which is a specific questionnaire to evaluate the quality of life and symptoms in patients with chronic venous disease.

Methods: The VEINES-QOLSym was adapted according to the recommended methodology and administered to 74 subjects (53,7 $\pm 12,3$ years-old) with chronic venous disease, who were assisted at the outpatient clinic.

Results: The results showed a good internal consistency for VEINES-QOL (Cronbach's alpha $(\alpha)=0,88)$ and VEINES/Sym $(\alpha=0,84)$. The inter and intra-rater reliability evaluated by the Intraclass Correlation Coefficient ranged from 0,95-0,98 for VEINES-QOL and 0,76-0,79 for the VEINES/Sym. The intraclass correlation coefficient was calculated for each item and some of them showed values lower than the expected, deserving a revision. The correlations with SF-36 were significant ( $p<0.001)$; however, of moderate magnitude. Only the VEINES-QOL was capable to distinguish the subjects in two groups, according to the severity of chronic venous disease $(p=0.02)$.

Conclusion: The VEINES-QOL/Sym, Brazil-Portuguese version has presented good clinimetric properties and has shown to be applicable to the population.
\end{abstract}

Keywords: Translating; psychometrics; questionnaire; venous insufficiency; quality of life.

\section{Introdução}

O avanço tecnológico e científico na sociedade atual possibilitou diagnosticar e tratar doenças de forma mais eficiente, aumentando a expectativa de vida e expondo a população a um risco de desenvolver doenças crônicodegenerativas ${ }^{1}$. Dentre as doenças crônicas que atingem a população adulta, pode-se destacar a doença venosa crônica (DVC), que é definida como uma disfunção no sistema venoso dos membros inferiores decorrente à hipertensão 
venosa, causada por incompetência valvular e/ou obstrução do fluxo venoso ${ }^{2}$.

As manifestações clínicas decorrentes da DVC são qualificadas com base na classificação clínica, etiológica, anatômica e patológica (CEAP, do inglês Clinical manifestations, Etiologic factors, Anatomic distribution of disease, Pathophysiologic findings). De acordo com esta classificação, os sinais clínicos são categorizados em sete classes: $\mathrm{C}_{0}$ - sinais de doença venosa não-visíveis e não-palpáveis; $\mathrm{C}_{1}$ - telangectasias ou veias reticulares; $\mathrm{C}_{2}$ - veias varicosas; $\mathrm{C}_{3}$ - edema; $\mathrm{C}_{4}$ - alterações da pele e tecido subcutâneo (4a - pigmentação ou eczema e $4 \mathrm{~b}$ - lipodermatoesclerose ou atrofia branca); $\mathrm{C}_{5}$ - alterações de pele com úlcera cicatrizada e $\mathrm{C}_{6}$ - alterações de pele com úlcera ativa ${ }^{3}$.

Estas manifestações clínicas associadas ou não aos sintomas como dor, câimbras, prurido e sensação de pernas pesadas e queimação são frequentemente associadas às limitações nas atividades diárias e no desempenho funcional, além de alterações psicológicas e mudanças na percepção do estado de saúde. Devido a estas repercussões, vários autores têm ressaltado a importância da inclusão da avaliação da qualidade de vida (QV) à abordagem de pacientes com $\mathrm{DVC}^{4-7}$.

Segundo o World Health Organization Quality of Life Group (WHOQOL Group), a QV é definida como a percepção do indivíduo de sua posição na vida, no contexto da cultura e sistemas de valores nos quais vive e em relação aos seus objetivos, expectativas, padrões e preocupações ${ }^{8}$. Este é um conceito amplo e complexo que envolve saúde física, estado psicológico, nível de independência, relações sociais, crenças pessoais e a relação com as características do ambiente ${ }^{9-11}$.

$\mathrm{O}$ Venous Insufficiency Epidemiological and Economic Study (VEINES) foi um estudo multicêntrico que objetivou avaliar os resultados clínicos, a QV, os custos e o uso de serviços de saúde de indivíduos com $\mathrm{DVC}^{12}$. Em virtude de existirem poucos questionários específicos para avaliar a QV em pacientes com DVC, e a maioria deles negligenciar a avaliação do impacto funcional, psicológico e social da doença, os autores desenvolveram um instrumento específico para avaliação da QV na DVC, o Venous Insufficiency Epidemiological and Economic Study - Quality of life/Symptom (VEINES-QOL/Sym). O formato dos itens, seu conteúdo e a escala de respostas foram elaborados com base no Medical Outcomes Study Short-form 36 (SF-36), que é um instrumento genérico para avaliação da QV. O VEINES-QOL/Sym foi validado em quatro países (Canadá, Bélgica, França e Itália) e possui versões em inglês, francês, francês canadense e italiano ${ }^{4,12}$.

O VEINES-QOL/Sym, na versão original, consiste em um questionário autoaplicável com 26 itens: dez relacionados aos sintomas (questões 1 e 7 ), nove ao desempenho das atividades de vida diária (questões 4, 5 e 6), um ao horário do dia que os sintomas são mais intensos (questão 2), um às alterações ocorridas com relação à doença no último ano (questão 3) e cinco ao impacto psicológico (questão 8). O tempo considerado para as questões relacionadas aos sintomas, às limitações na vida diária e ao impacto psicológico compreende as últimas quatro semanas ${ }^{4}$.

O VEINES-QOL/Sym produz dois escores, sendo um a estimativa do impacto da DVC na QV, o VEINES-QOL, e outro relacionado aos sintomas decorrentes da DVC, o VEINES-Sym. Para obtenção do escore total do VEINESQOL, devem ser considerados os 25 itens que compreendem as questões $1,3,4,5,6,7$ e 8 do questionário, a questão 2 deve ser relacionada ao horário do dia em que os sintomas são mais intensos, e não deve ser utilizada para cálculo do escore, fornecendo apenas informação descritiva ${ }^{4}$.

O escore produzido pelo VEINES-Sym inclui dez itens (questões 1 e 7). Destes, nove estão relacionados aos sintomas: pernas pesadas, pernas doloridas, inchaço, câimbras noturnas, sensação de calor ou queimação, pernas inquietas, latejamento, coceira e sensação de formigamento. Tais sintomas são avaliados de acordo com a frequência, utilizando uma escala tipo Likert de cinco pontos. O outro item, a questão 7 , está relacionado à dor nas pernas e é avaliado quanto à intensidade por uma escala tipo Likert de seis pontos ${ }^{4}$.

Inicialmente, para o cálculo dos escores do VEINESQOL e do VEINES-Sym, as seguintes etapas devem ser seguidas: recodificação das questões 3, 6 e 7 (inversão do valor dos escores); cálculo da média e do desvio padrão de cada item; padronização de cada item em um escore $\mathrm{Z}$ de média zero e desvio padrão igual a um, e cálculo do valor do escore do indivíduo menos a média da questão dividida pelo desvio padrão. O escore total é calculado como a média dos escores $\mathrm{Z}$ dos itens envolvidos neste. O escore final é transformado de modo a apresentar média igual a 50 e desvio padrão de 10, multiplicando-se o escore total por $10 \mathrm{e}$ adicionando 50 ao resultado. Se menos de $50 \%$ dos 25 itens incluídos no escore VEINES-QOL não são respondidos, o escore final não é calculado, e a resposta do indivíduo é considerada ausente. Da mesma forma, se há menos que 50\% dos dez itens respondidos, o escore VEINES/Sym não é calculado. Tanto para o VEINES-QOL quanto para o VEINE/Sym, escores maiores indicam melhores desfechos ${ }^{4,13}$.

O instrumento, na versão original, apresenta boas propriedades clinimétricas além de uma metodologia objetiva, não-onerosa, praticável em qualquer recinto, que completa a avaliação clínica convencional ${ }^{4}$. 
Os questionários da QV são criados com base na realidade sociocultural do país de origem, e sua utilização em populações com características socioculturais diferentes requer tradução criteriosa e avaliação da necessidade de adaptações culturais $^{14-17}$. A versão original do questionário VEINESQOL/Sym foi desenvolvida na língua inglesa e para sua aplicação em outras populações, é necessário que, além da tradução, seja verificada a necessidade de adaptação transcultural para a população-alvo e a avaliação das propriedades clinimétricas, visto que apenas a tradução literal do instrumento não é o bastante para sua utilização em outros idiomas ${ }^{16,17}$.

O objetivo do presente estudo foi traduzir, adaptar culturalmente o VEINES-QOLSym e analisar as propriedades clinimétricas da versão em português-Brasil do questionário.

\section{Metodologia}

Trata-se de um estudo metodológico, de corte transversal, aprovado pelo Comitê de Ética em Pesquisa do Hospital Municipal Odilon Behrens, onde foi realizado (Parecer ETIC 83/2006). Os participantes assinaram o termo de consentimento livre e esclarecido.

\section{Procedimentos}

Segundo recomendação de Beaton et al. ${ }^{16}$, inicialmente o VEINES-QOL/Sym foi traduzido, do ponto de vista semântico, idiomático, cultural e conceitual, para a língua portuguesa-Brasil, por duas tradutoras bilíngues, cujo idioma de origem era o português. Uma síntese das duas versões traduzidas foi realizada a partir da reunião das tradutoras com objetivo de se chegar a uma única versão final. A partir desta, foi realizada a retrotradução, de forma independente, por dois outros tradutores qualificados, residentes no Brasil, cuja língua de origem é o inglês (língua do instrumento original) ${ }^{16}$.

Um comitê de juízes especialistas foi constituído de profissionais da área com domínio do tema e fluência nos dois idiomas, para a produção da versão final. O comitê discutiu a equivalência linguística e idiomática entre a versão traduzida, as duas versões retrotraduzidas e a versão original e não achou necessária a eliminação de qualquer item, mas julgou necessária a inclusão da atividade "ir à igreja" nos exemplos do item "d" da questão 4. Para a questão 1, sugeriu-se acrescentar a informação ao item 'pernas inquietas': "Não acho posição para minhas pernas". Outra modificação, recomendada pelo comitê, foi que o instrumento fosse aplicado por meio de entrevista e não autoadministrado como na sua versão original. Esta recomendação foi sugerida em face da possibilidade de parte da população avaliada ser analfabeta ou semianalfabeta.

Após aprovação da versão final do instrumento pelo comitê, a técnica de prova recomendada por Beaton et al. ${ }^{16}$ foi realizada por meio da aplicação do VEINES-QOL/Sym, versão português-Brasil, em 30 indivíduos adultos da comunidade geral, com diagnóstico de DVC, sem alterações cognitivas que pudessem interferir na compreensão do questionário. Como não houve nenhum problema ou dúvidas relacionadas à redação ou clareza dos itens e respostas, a versão elaborada pelo comitê de juízes foi mantida ${ }^{16}$ (Anexo 1).

\section{Aplicação do VEINES-QOL/Sym, versão português-Brasil}

Foram selecionados indivíduos com diagnóstico de DVC, sem restrição quanto ao sexo, idade ou estadiamento da doença, os quais frequentaram um ambulatório de cirurgia vascular de um hospital da rede pública durante o período de realização da pesquisa. Foram excluídos aqueles que apresentavam diagnóstico médico de insuficiência renal crônica, insuficiência cardíaca congestiva descompensada, doença pulmonar obstrutiva crônica, comprometimento de raiz nervosa lombar, doença arterial periférica de membros inferiores (índice tornozelo braço menor que 0,9 ), osteoartrite de uma ou mais articulações dos membros inferiores e déficits de compreensão e fala que impedissem sua participação.

Os indivíduos foram esclarecidos com relação aos objetivos da pesquisa e convidados a assinarem o termo do consentimento livre e esclarecido. Em seguida, foi realizada uma entrevista para coleta dos dados sociodemográficos e clínicos.

Para caracterizar os sinais clínicos da DVC, utilizou-se a classificação CEAP. Os membros inferiores foram examinados, estando os mesmos na posição ortostática, por inspeção visual, sempre por um mesmo examinador treinado. No caso dos dois membros inferiores serem acometidos, foi considerado o de maior escore da CEAP. Os membros classificados como 4a (pigmentação ou eczema) e 4b (lipodermatoesclerose ou atrofia branca) foram agrupados em um mesmo grupo (Classe 4$)^{3}$.

Os questionários VEINES-QOL/Sym, versão português-Brasil, e o SF-36 ${ }^{18}$ foram administrados na forma de entrevista, por dois examinadores treinados que seguiram instruções padronizadas.

\section{Análise das propriedades clinimétricas}

A confiabilidade do VEINES-QOL/Sym, versão Português-Brasil, foi avaliada pela análise da consistência interna e da confiabilidade interexaminadores e intraexaminador. 
A consistência interna se refere à extensão na qual os itens que compreendem uma determinada escala medem um mesmo construto $^{14,19}$, e foi analisada pelo alfa de Cronbach $(\alpha)$, sendo considerados valores aceitáveis de $\alpha$ entre 0,70 e $0,90^{19}$.

A confiabilidade intra- e interexaminadores foi avaliada mediante aplicação do questionário por dois examinadores previamente treinados, em dois momentos distintos com intervalo de tempo entre uma entrevista e outra de no mínimo sete dias e no máximo dez dias, em 12 participantes. No primeiro momento, o indivíduo foi avaliado pelo examinador A e, no segundo, a avaliação foi repetida pelo examinador A e aplicada também pelo examinador $\mathrm{B}$. O coeficiente de correlação intraclasse (CCI) foi utilizado para investigar a confiabilidade intra- e interexaminadores ${ }^{19}$.

A validade convergente indica que medidas de uma escala estão correlacionadas com outra que avalia o mesmo construto ou construto similar, foi avaliada pela análise de correlação (correlação de Pearson) entre os escores do VEINES-QOL/ Sym versão Português-Brasil e os do questionário SF-36 ${ }^{18}$. Tal questionário é composto de 36 questões agrupadas em oito domínios: quatro relacionados à saúde física (capacidade funcional, aspecto físico, dor e estado geral da saúde) e quatro à saúde mental (vitalidade, aspecto social, aspecto emocional e saúde mental). Apresenta um escore final que varia de zero a 100 , sendo que, quanto maior o escore, melhor a percepção que o indivíduo tem em relação à sua QV. A média de escores dos domínios relacionados à saúde física representou o escore do componente de saúde física (CSF), e a média dos escores dos domínios relacionados à saúde mental representou o escore do componente de saúde mental (CSM) ${ }^{18}$.

A validade de grupos conhecidos indica a capacidade do instrumento em discriminar indivíduos que sabidamente apresentam características diferentes. Por meio de um teste $t$ para amostras independentes, foram comparadas as médias dos escores obtidos no VEINES-QOL e no VEINES/Sym entre dois grupos formados, segundo a variável CEAP, sendo um grupo formado pelos participantes CEAP 1, 2 e 3 (menos comprometidos clinicamente em relação à DVC) e o outro grupo CEAP 4, 5 e 6 (mais comprometidos clinicamente em relação à DVC) ${ }^{19}$.

Os dados foram expressos em média \pm desvio padrão, valores mínimos e máximos e em porcentagem. Foi considerada diferença estatisticamente significativa quando $\alpha<5 \%{ }^{19}$. O pacote estatístico utilizado na análise foi o Statistical Package for the Social Sciences (SPSS), versão 16.0 para Windows ${ }^{\circledast}$.

\section{Resultados}

Foram avaliados 74 indivíduos com média de idade de $53,7 \pm 12,3$ anos, dos quais $70,3 \%$ eram mulheres. Todos os participantes tinham algum grau de comprometimento venoso, sendo 48,7\% da amostra CEAP 5 e 6 . Os dados clínicos e sociodemográficos estão representados na Tabela 1.

Os resultados evidenciaram adequada consistência interna para VEINES-QOL $(\alpha=0,88)$ e VEINES/Sym $(\alpha=0,84)$ e adequada confiabilidade interexaminadores (VEINES-QOL - CCI: 0,95 e VEINES/Sym - CCI: 0,76) e intraexaminador (VEINES-QOL - CCI: 0,98 e VEINES/ Sym - CCI: 0,79). Quando foi avaliado o CCI de cada item separadamente, observou-se que poucos itens (1-8, 2, 3, 4-a e 5-c) apresentaram valores abaixo de 0,5 (Tabela 2).

A análise de correlação entre os escores do VEINESQOL e VEINE/Sym e dos CSF e CSM do SF-36 evidenciaram valores do coeficiente de correlação de Pearson $(r)$ de moderada magnitude ${ }^{19}$, como pode ser observado na Tabela 3. Houve significância estatística para todas as correlações avaliadas $(\mathrm{p}<0,001)$.

Tabela 1 - Dados sociodemográficos e clínicos

\begin{tabular}{|c|c|c|}
\hline Variáveis & $\mathrm{n}$ & $\%$ \\
\hline \multicolumn{3}{|l|}{ Sexo } \\
\hline Masculino & 22 & 30 \\
\hline Feminino & 52 & 70 \\
\hline \multicolumn{3}{|l|}{ Idade (anos) } \\
\hline Média土DP (mínimo-máximo) & $53,70 \pm 12,36(26-82)$ & \\
\hline \multicolumn{3}{|l|}{ Escolaridade (anos de estudo) } \\
\hline Sem escolaridade & 4 & 5,4 \\
\hline Entre 1 e 7 anos & 46 & 62,2 \\
\hline 8 ou mais & 24 & 32,4 \\
\hline \multicolumn{3}{|l|}{ Renda familiar } \\
\hline Menos de $R \$ 496,00$ & 33 & 44 \\
\hline Entre $R \$ 497,00$ e 1.064,00 & 30 & 41 \\
\hline Entre $R \$ 1065,00$ e $R \$ 2943,00$ & 11 & 15 \\
\hline \multicolumn{3}{|l|}{ Duração da doença (anos) } \\
\hline Média_DP (mínimo-máximo) & $16,65 \pm 12,33(1-50)$ & \\
\hline \multicolumn{3}{|l|}{ Classificação CEAP } \\
\hline 1 & 3 & 4,1 \\
\hline 2 & 6 & 8,0 \\
\hline 3 & 10 & 13,5 \\
\hline 4 & 19 & 25,7 \\
\hline 5 & 19 & 25,7 \\
\hline 6 & 17 & 23 \\
\hline \multicolumn{3}{|l|}{ Doenças associadas } \\
\hline HAS & 27 & 36,5 \\
\hline HAS + outra doença & 10 & 13,5 \\
\hline Nenhuma & 34 & 45,9 \\
\hline Outras & 3 & 4,1 \\
\hline \multicolumn{3}{|l|}{ História familiar de DVC } \\
\hline $\operatorname{sim}$ & 52 & 70,2 \\
\hline Não & 11 & 14,9 \\
\hline Não souberam responder & 11 & 14,9 \\
\hline
\end{tabular}

DP: desvio padrão; HAS: hipertensão arterial sistêmica; DVC: doença venosa crônica. 
Foram encontradas diferenças significativas entre os grupos CEAP 1, 2 e 3 e CEAP 4, 5 e 6 apenas para o escore do VEINES-QOL $(\mathrm{p}=0,02)$. Para o VEINES/Sym, o valor de $\mathrm{p}$ foi de 0,50 . Tanto para VEINES-QOL quanto para o VEINES/Sym, os escores diminuem à medida que a gravidade da DVC aumenta, representando um declínio na QV com o agravamento da DVC. Porém, apenas o escore produzido pelo VEINES-QOL conseguiu diferenciar a QV de

Tabela 2 - Confiabilidade interexaminadores e intraexaminador do VEINES-QOL/Sym, versão português, Brasil

\begin{tabular}{|c|c|c|}
\hline \multirow[t]{2}{*}{ Itens } & $\begin{array}{c}\text { Confiabilidade } \\
\text { interexaminadores }\end{array}$ & $\begin{array}{l}\text { Confiabilidade } \\
\text { intraexaminador }\end{array}$ \\
\hline & ICC & ICC \\
\hline Q 1-1 & 0,78 & 0,91 \\
\hline Q 1-2 & 0,61 & 0,65 \\
\hline Q 1-3 & 0,91 & 0,95 \\
\hline Q 1-4 & 0,53 & 0,67 \\
\hline Q 1-5 & 0,75 & 0,89 \\
\hline Q 1-6 & 1,00 & 1,00 \\
\hline Q 1-7 & 0,89 & 0,92 \\
\hline Q 1-8 & 0,29 & 0,37 \\
\hline Q 1-9 & 0,55 & 0,69 \\
\hline Q2 & 0,25 & 0,27 \\
\hline Q 3 & 0,45 & 0,68 \\
\hline Q 4a & 0,42 & 0,44 \\
\hline Q 4b & 0,80 & 0,88 \\
\hline Q 4C & 0,85 & 0,86 \\
\hline Q 4d & 0,65 & 0,68 \\
\hline Q 5a & 1,00 & 1,00 \\
\hline Q 5b & 0,65 & 0,78 \\
\hline Q 5c & 0,00 & 0,00 \\
\hline Q 5d & 0,65 & 0,78 \\
\hline Q6 & 0,92 & 0,96 \\
\hline Q7 & 0,83 & 0,98 \\
\hline Q 8a & 0,79 & 0,86 \\
\hline Q 8b & 0,83 & 0,92 \\
\hline Q 8c & 0,54 & 0,71 \\
\hline Q 8d & 0,94 & 0,96 \\
\hline Q 8e & 0,67 & 0,87 \\
\hline VEINES-QOL & 0,95 & 0,98 \\
\hline VEINES/Sym & 0,76 & 0,79 \\
\hline
\end{tabular}

ICC: coeficiente de correlação intraclasse.

Tabela 3 - Análise da validade convergente do VEINES-QOL/Sym, versão português, Brasil

\begin{tabular}{lcc}
\hline & \multicolumn{2}{c}{ Coeficiente de correlação de Pearson $(r)$} \\
\cline { 2 - 3 } & VEINES-QOL & VEINES/Sym \\
\hline CSF do SF-36 & $r=0,65$ & $r=0,51$ \\
CSM do SF-36 & $r=0,52$ & $r=0,49$ \\
\hline
\end{tabular}

CSF: componente de saúde física; CSM: componente de saúde mental. forma estatisticamente significativa entre os Grupos CEAP 1,2 e 3 e CEAP 4,5 e 6.

O tempo gasto para aplicação do VEINES-QOLSym versão português-Brasil na população estudada foi de aproximadamente dez minutos.

\section{Discussão}

Não foram necessárias importantes adequações da estrutura original dos itens do VEINES-QOL/Sym, pelo fato de seus itens serem plenamente aplicáveis à cultura brasileira. Em virtude das altas taxas de analfabetismo em nossa população e para padronizar a forma de aplicação, optou-se por administrá-lo na forma de entrevista diferente da forma como foi administrada a versão original (autoadministrado) ${ }^{4}$. As formas de administração de questionários de QV mais frequentes são a autoaplicação e a entrevista ${ }^{8,9}$. Wu et al. ${ }^{20}$ investigaram se haveria diferença entre os escores de instrumentos de QV administrados mediante entrevista e autoaplicados, em amostras de pessoas soropositivas, e não observaram diferença significativa entre os escores nas duas modalidades de aplicação ${ }^{20}$.

Os valores de $\alpha$ de Crombach encontrados, referentes à análise da consistência interna VEINES-QOL, $\alpha=0,88$, e VEINES-Sym, $\alpha=0,84$ foram elevados, indicando que as escalas apresentam adequada consistência interna. Estes resultados são semelhantes aos encontrados na versão original do instrumento VEINES-QOL, $\alpha=0,91$, e VEINES-Sym, $\alpha=0,87^{4} \mathrm{e}$ em outros estudos que também avaliaram tal propriedade VEINES-QOL, $\alpha=0,91$, e VEINES-Sym, $\alpha=0,88,{ }^{13}$ e VEINES-QOL, $\alpha=0,91$, e VEINES-Sym, $\alpha=0,88^{21}$.

As confiabilidades intra e interexaminadores se mostraram adequadas para o escore total do VEINES-QOL e VEINES-Sym, o que garante que o instrumento é efetivo para obter resultados fidedignos na população. Os resultados evidenciaram maior confiabilidade intraexaminador do que interexaminadores tanto para o escore total quanto para itens, o que concorda com estudos anteriores que também avaliaram a confiabilidade do instrumento ${ }^{4,13,21}$.

Quando os itens das escalas foram avaliados separadamente, os valores do CCI encontrados para as questões 1-8, 2, 3, 4-a e 5-c foram abaixo de 0,5 tanto para a confiabilidade intraexaminador quanto para interexaminadores (exceto questão 3 para avaliação da confiabilidade intraexaminador). Futuramente, tais questões devem ser revistas na tentativa de verificar os motivos de instabilidade dos indicadores apontados e melhorar a qualidade geral do instrumento.

O SF-36 é muito utilizado em pesquisas que envolvem avaliação de QV na DVC $5,6,7,11,22$. Apesar de instrumentos 
genéricos, como o SF-36, refletirem o impacto das doenças sobre aspectos gerais da saúde e terem a vantagem de permitir comparação de indivíduos com diferentes doenças, eles são menos sensíveis para explorar os efeitos específicos da doença na $\mathrm{QV}^{9,10}$. A recomendação vigente para avaliar a QV, como desfecho primário, é de que seja usado um instrumento específico associado a um genérico ${ }^{8-10}$.

Os resultados das análises de correlação entre o VEINES-QOL/Sym e o SF-36 apresentaram valores de $r$ em torno de $0,5^{19}$. Considerando que os dois questionários medem construtos semelhantes e que um é específico e o outro é genérico, correlações de moderada magnitude são esperadas, visto que se os valores de $r$ encontrados fossem muito altos (próximos de um), poderia significar que ambos são redundantes; caso os valores de $r$ encontrados fossem muito baixos, poderia significar que não existem evidências de que as escalas medem construtos semelhantes ${ }^{13}$. Observouse que o valor de $r$ foi maior entre o VEINES-QOL e o CSF $(\mathrm{r}=0,65)$ do que entre o VEINES-QOL e o CSM $(\mathrm{r}=0,52)$. Isto pode ser explicado pelo fato de o VEINES-QOL apresentar mais questões relacionadas aos aspectos físicos e funcionais. Resultados semelhantes foram observados em estudos prévios que avaliaram as propriedades clinimétricas do VEINES-QOL/Sym ${ }^{4,13,22}$.

As médias dos escores produzidos pelo VEINES-QOL foram significativamente menores $(\mathrm{p}=0,02)$ para os indivíduos do Grupo CEAP 4, 5 e 6 em relação ao Grupo CEAP 1,2 e 3, demonstrando que o VEINES-QOL foi capaz de detectar diferenças relacionadas à QV em dois grupos com características clínicas distintas. Vários autores demonstraram a existência de uma relação direta entre a gravidade da DVC e QV, sobretudo em relação aos aspectos físicos e funcionais ${ }^{4,7,11,22}$. Notou-se redução do escore do VEINES/ Sym com o agravamento da doença, porém sem significância estatística $(\mathrm{p}=0,50)$. Estudos que avaliaram a relação dos sintomas e a gravidade da DVC demonstraram que mesmo na presença de telangectasias e veias reticulares, sintomas como latejamento, sensação de calor nas pernas e dor podem estar presentes sem gerar na mesma proporção limitação física e funcional, como ocorre nas fases mais avançadas da doença, o que pode explicar o fato de o VEINES/Sym não ter discriminado os grupos ${ }^{7,22}$.

\section{Conclusão}

O VEINES-QOL/Sym versão português-Brasil mostrou ser um instrumento adequado do ponto de vista semântico e linguístico, com boas propriedades clinimétricas e de fácil aplicação. Vale ressaltar a necessidade de mais análises relacionadas à confiabilidade, sobretudo dos itens que apresentaram valores de ICC abaixo de 0,50. Este instrumento poderá contribuir para o desenvolvimento de novas pesquisas e para melhor compreensão dos aspectos da QV mais afetados pela DVC.

\section{Referências}

1. Martins LM, França, APD, Kimura M. Qualidade de vida de pessoas com doença crônica. Rev. Latino-am. Enfermagem. 1996;4:5-18.

2. Porter MJ, Moneta GL. Reporting standards in venous disease: An update. International Consensus Committee on Chronic Venous Disease. J Vasc Surg. 1995; 21:635-45.

3. Eklöf B, Rutherford RB, Bergan JJ, Carpentier PH, Gloviczki P, Kistner RL, Meissner MH, Moneta GL, Myers K, Padberg FT, Perrin M, Ruckley CV, Smith PC, Wakefield TW; American Venous Forum International Ad Hoc Committee for Revision of the CEAP Classification. Revision of the CEAP classification for chronic venous disorders: Consensus statement. J Vasc Surg. 2004;40: 1248-52.

4. Lamping DL, Schroter S, Kurz X, Kahn SR, Abenhaim L. Evaluation of outcomes in chronic venous disorders of leg: development of a scientifically rigorous, patient-reported measure of symptoms and quality of life. J Vasc Surg. 2003;37(2):410-9.

5. van Korlaar I, Vossen C, Rosendaal F, Cameron L, Bovil E, Kaptein A. Quality of life in venous disease. Thromb Haemost. 2003;90(1): 27-35.

6. Lamping DL. Measuring health-related quality of life in venous diseases: practical and scientific considerations. Angiology. 1997;48(1):51-7.

7. Kurz X, Lamping DL, Kahn SR, Baccaglini U, Zuccarelli F, Spreafico G, Abenhaim L; VEINES Study Group. Do varicose veins affect quality of life? Results of an international population-based study. J Vasc Surg. 2001;34(4):641-8.

8. WHOQOL GROUP. The World Health Organization quality of life assessment (WHOQOL): position paper from the World Health Organization. Soc Sci Med. 1995;41(10):1403-9.

9. Seidl EMF, Zannon CMLC. Qualidade de vida e saúde: aspectos conceituais e metodológicos. Cad Saúde Pública. 2004;20:580- 8.

10. Ciconelli RM. Medidas de avaliação de qualidade de vida. Rev Bras Reumatol. 2003;43:9-13.

11. Kaplan RM, Criqui M, Denenberg JO, Bergan J, Fronek A. Quality of life in patients with chronic venous disease San Diego population study. J Vasc Surg. 2003;37(5):1047-53

12. Abenhaim L, Kurz X. The VEINES Study (VEnous INsufficiency Epidemiologic and Economic Study): An International Cohort Study on Chronic Venous Disorders of the leg. Angiology. 1997;48(1):59-66.

13. Kahn SR, Lamping DL, Ducruet T, Arsenault L, Miron MJ, Roussin A, Desmarais S, Joyal F, Kassis J, Solymoss S, Desjardins L, Johri M, Shrier I; VETO Study investigators. VEINES-QOL/Sym questionnaire was a reliable and valid disease-specific quality of life measure for deep venous thrombosis. J Clin Epidemiol. 2006;59(10): 1049-56.

14. Gadotti IC, Vieira ER, Magee DJ. Importance and clarification of measurement properties in rehabilitation. Rev Bras Fisioter. 2006;10 (2):137-46 
15. Guillemin F, Bombardier C, Beaton D. Cross-cultural adaptation of health related quality of life measures: literature review and proposed guidelines. J Clin Epidemiol. 1993;46(12):1417-32.

16. Beaton DE, Bombardier C, Guillemin F, Ferraz MB. Guidelines for the process of cross-cultural adaptation of self-report measures. Spine. 2000;35(24):3186-91.

17. Maher CG, Latimer J, Costa LOP. The relevance of cross-cultural adaptation and clinimetrics for physical therapy instruments. Rev Bras Fisioter. 2007;11(4):245-52.

18. Ciconelli RM, Ferraz MB, Santos W, Meinão I, Quaresma MR. Tradução para a língua portuguesa e validação do questionário genérico de avaliação de qualidade de vida SF-36 (Brasil SF-36). Rev Bras Reumatol. 1999;39:143-50.

19. Portney LG, Watkins MP. Foundations of clinical research applications to practice. $3^{a}$ ed. New Jersey: Pearson Prentice Hall; 2008.

20. Wu AW, Hays RD, Kelly S, Malitz F, Bozzette SA. Applications of the Medical Outcomes Study health-related quality of life measures in HIV/AIDS. Qual Life Res. 1997;6(6):531-54.

21. Eden T, Garrat AM, Klow NE, Sandset PM. Assessing burden of illness following acute deep vein thrombosis: data quality, reliability and validity of the Norwegian version of VEINES-QOL/ Sym, a disease-specific questionnaire. Scand J Caring Sci. 2009;23(2): 369-74.

22. Kahn SR, M'lan CE, Lamping DL, Kurz X, Bérard A, Abenhaim LA; VEINES Study Group. Relationship between clinical classification of chronic venous disease and patient-reported quality of life: Results from an international cohort study. J Vasc Surg. 2004;39(4):823-8.

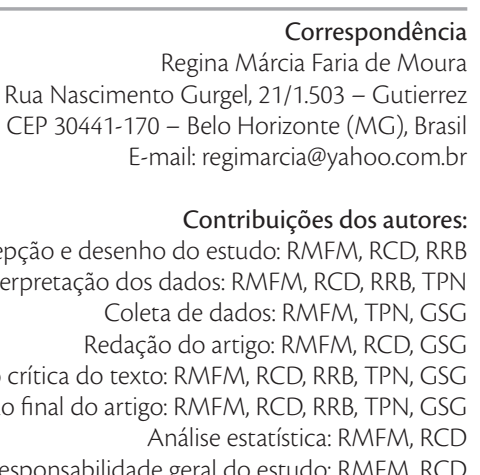

